\title{
Failure Factors Quality in Vocational and Technical Education in Nigeria Higher Institutions: The Use of Rasch Model
}

\author{
Olojuolawe Sunday Rufus, Tunde Ajayi Babatunde Joseph, Awolumate Emmanuel Olorunfemi
}

\begin{abstract}
The study is a case study of the student academic performance in vocational and technical education at the College of Education, Ikere Ekiti. The academic performance of students has been abysmally low. This has grossly impacted the students' enrolment in the departments and the College in general. The paper identifies the major causes of the failures in vocational and technical education courses in the College. The survey research employing exploratory sequential mixed method was used. It involves 12 Participants and 50 respondents. The Nvivo12 software was used to obtain the word cloud. The Rasch Analysis Model was applied to obtain the Person and Item separations, reliabilities and the respondent's agreement. The hierarchy details the factors in order relativity and difficulty level. Findings shows that both internal and external factors are the main sources of students' failure.

Keywords: Technical Education, Technopreneur, Graduate,
\end{abstract} Employability

\section{INTRODUCTION}

The introduction of vocational and technical education into the curriculum of Colleges of education in Nigeria was aimed at inculcating saleable skills into the youths [1]-[4]. The recipients are trained to acquire skills, attitude and knowledge which are needed for the economic growth of the nation (Kehinde \&Adewuyi, 2015). The general aim was to promote human resource development, productivity and economic development. Vocational and technical education is crucial to national development of most countries of the world [5]. The poor image of vocational and technical education have been linked to the rising rate of youth unemployment in Nigeria [6]-[9].Despite the strong commitment of government at providing adequate infrastructures and facilities in Nigeria tertiary institutions and Colleges of Education in particular, vocational and technical education is still faced with myriad of problems [9]-[11]. These ranges from the classrooms to the labour market. Studies conducted by Hayatu \& Abubakar, (2019)

Revised Manuscript Received on May 19, 2020.

* Correspondence Author

Olojuolawe Sunday Rufus*, Technical Education Department, College of Education, Ikere-Ekiti, Nigeria. Email: olojuolawerufus4@gmail.com

Mr. Tunde Ajayi Babatunde Joseph, Fine and Applied Arts department, College of Education, Ikere-Ekiti, Nigeria. Email: ajayi.babatunde@coeikere.edu.ng

Mr Awolumate Emmanuel Olorunfemi, Emmanuel, Technical Education Department, College of Education, Ikere-Ekiti, Nigeria. awoluolorufemi@gmail.com

(C) The Authors. Published by Blue Eyes Intelligence Engineering and Sciences Publication (BEIESP). This is an open access article under the CC BY-NC-ND license (http://creativecommons.org/licenses/by-nc-nd/4.0/) indicated external and external factors as the main contributing factors for students' academic poor performance in Nigeria Higher institutions. Internal factors are students induced factors. While the teachers, schools, government and parental factors are external factors [12], [13]. Recently, the rate of mass failure in vocational and technical education attracted the attention of the College Management and ordered a probe. Consequently, this study is aimed at identifying the major causes of students' failure in TVET. The specific objective includes identifying the problems, order the problems into the level of severity and offering possible suggestion. Figure 1.1 shows the conceptual framework of the study.

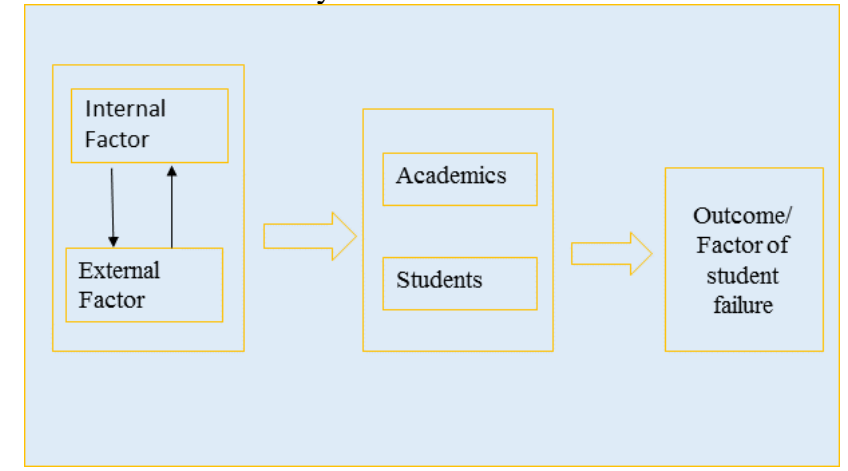

Figure 1.1: Conceptual framework

\section{METHODOLOGY}

This study employs exploratory sequential mixed methods because of the large population [14], [15]. The study consists of personal interview with the Academics and the students in the school of study. They forms the major critical stakeholders for the study. Table 1.1 shows the list of the departments in the school, including the number of Academics and students.

Table 1.1: Number of Departments

\begin{tabular}{|l|l|l|l|}
\hline S/N & DEPARTMENT & ACADEMICS & STUDENTS \\
\hline 1 & $\begin{array}{l}\text { Agricultural } \\
\text { Education }\end{array}$ & & \\
\hline 2 & $\begin{array}{l}\text { Business } \\
\text { Education }\end{array}$ & & \\
\hline 3 & $\begin{array}{l}\text { Fine \& Applied } \\
\text { Arts Education }\end{array}$ & & \\
\hline 4 & $\begin{array}{l}\text { Home Economics } \\
\text { Education }\end{array}$ & & \\
\hline 5 & $\begin{array}{l}\text { Technical } \\
\text { Education }\end{array}$ & & \\
\hline
\end{tabular}


Failure Factors Quality in Vocational and Technical Education in Nigeria Higher Institutions: The Use of Rasch Model

The population for the study is made up of both Academics and students in the school of vocational and technical education in the college. The purposive sampling technique was used for the qualitative aspect of the study because of the time frame [16], [17]. Consequently, the sample size for the qualitative study involves 10 participants which included 5 academics and 5 students.

Since the current study is seeking to establish the validity of the factors responsible for students' failure in vocational and technical education in Ikere College of Education using the Rasch Analysis Model, it is therefore important to follow the sample size prescribed for Rasch Measurement Model. The researcher decided to go for \pm 1 logit with a $99 \%$ confidence level, and the sample size for most purposes is 50 . Rasch analysis sample size of at least 50 respondents and a minimum of 20 items are suggested for attaining stable indices [18], [19].

\section{DATA ANALYSIS}

Table 1.2 shows the data analysis for student factors.

Table 1.2: Interview Findings for Student Factors.

\begin{tabular}{|c|c|c|c|}
\hline $\begin{array}{l}\text { Example of } \\
\text { responses }\end{array}$ & Respondents & Codes & Themes \\
\hline $\begin{array}{l}\text { Most } \\
\text { students } \\
\text { don't attend } \\
\text { lectures...fi } \\
\text { rst lecture } \\
\text { only } 9 \text { came } \\
\text { for class out } \\
\text { of } 19\end{array}$ & AC1 AC2 & $\begin{array}{l}\text { Atten } \\
\text { dance } \\
\text { at } \\
\text { lectur } \\
\text { es }\end{array}$ & Absenteeism \\
\hline $\begin{array}{l}\text { Parents } \\
\text { poor } \\
\text { financial } \\
\text { background } \\
\text { delayed } \\
\text { registration } \\
\text {...poor } \\
\text { financial } \\
\text { status of the } \\
\text { students... a } \\
\text { bsence of } \\
\text { subsidy / } \\
\text { scholarship } \\
\text { s to cushion } \\
\text { the effects o } \\
\text { the high } \\
\text { school fees }\end{array}$ & $\begin{array}{l}\text { AC1 AC2 } \\
\text { AC3 ST1 } \\
\text { ST2 ST3 }\end{array}$ & $\begin{array}{l}\text { Late } \\
\text { registr } \\
\text { ation } \\
\text { for } \\
\text { cours } \\
\text { es }\end{array}$ & High tuition \\
\hline $\begin{array}{l}\text { Economic } \\
\text { situation in } \\
\text { the country } \\
\text {...lack of } \\
\text { job } \\
\text { opportuniti } \\
\text { es dampens } \\
\text { students } \\
\text { morale...gr } \\
\text { aduated } \\
\text { seniors } \\
\text { didn't have } \\
\text { jobs. }\end{array}$ & $\begin{array}{l}\text { AC1 AC2 } \\
\text { ST2 ST3 }\end{array}$ & $\begin{array}{l}\text { Weak } \\
\text { ened } \\
\text { intere } \\
\text { st }\end{array}$ & Motivation \\
\hline
\end{tabular}

\begin{tabular}{|c|c|c|c|}
\hline $\begin{array}{l}\text { Nothing to } \\
\text { arouse their } \\
\text { interest...la } \\
\text { ck of job } \\
\text { motivation. }\end{array}$ & & & \\
\hline $\begin{array}{l}\text { Most } \\
\text { students } \\
\text { failed to } \\
\text { participate } \\
\text { in tests, } \\
\text { assignment } \\
\text { s...care } \\
\text { free } \\
\text { attitudes } \\
\text { are } \\
\text { exhibited. } \\
\text { Lack of } \\
\text { interest in } \\
\text { education } \\
\text { because } \\
\text { there is no } \\
\text { job. Most } \\
\text { lecturers } \\
\text { are hostile. }\end{array}$ & $\begin{array}{l}\text { AC2 AC3 } \\
\text { ST1 ST2 ST3 }\end{array}$ & $\begin{array}{l}\text { Uncar } \\
\text { ed } \\
\text { attitud } \\
\text { es }\end{array}$ & Attitudes \\
\hline $\begin{array}{l}\text { Instructiona } \\
\text { l material } \\
\text { are not } \\
\text { available... } \\
\text { abstract } \\
\text { teaching... } \\
\text { absence of } \\
\text { teaching } \\
\text { materials }\end{array}$ & $\begin{array}{l}\text { AC1 AC2 } \\
\text { AC3 ST1 }\end{array}$ & $\begin{array}{l}\begin{array}{l}\text { Lack } \\
\text { of }\end{array} \\
\text { materi } \\
\text { als for } \\
\text { works } \\
\text { hop } \\
\text { practi } \\
\text { ce }\end{array}$ & $\begin{array}{l}\text { Abstract } \\
\text { teaching }\end{array}$ \\
\hline
\end{tabular}

Table 1.3: Summary of interview finding for Students factors

\begin{tabular}{|c|c|c|c|c|c|c|c|c|c|c|}
\hline \multirow{4}{*}{$\begin{array}{l}\text { Sub } \\
\text { Construct }\end{array}$} & \multicolumn{5}{|c|}{ Academics } & \multicolumn{5}{|c|}{ Students } \\
\hline & $\mathrm{A}$ & A & A & $\mathrm{A}$ & A & $S$ & $S$ & $S$ & $\mathrm{~S}$ & S \\
\hline & $\mathrm{C}$ & $\mathrm{C} 2$ & $\mathrm{C}$ & $\mathrm{C}$ & C5 & $\mathrm{T}$ & $\mathrm{T}$ & $\mathrm{T}$ & $\mathrm{T}$ & $\mathrm{T}$ \\
\hline & 1 & & 3 & 4 & & 1 & 2 & 3 & 4 & 5 \\
\hline Absenteeism & $*$ & $*$ & & & & & & & & \\
\hline High tuition & * & $*$ & * & & & $*$ & $*$ & $*$ & & \\
\hline Motivation & $*$ & $*$ & & & & & $*$ & $*$ & & \\
\hline Attitudes & & $*$ & $*$ & & & $*$ & $*$ & $*$ & & \\
\hline $\begin{array}{l}\text { Abstract } \\
\text { teaching }\end{array}$ & $*$ & $*$ & $*$ & & & $*$ & & & & \\
\hline
\end{tabular}

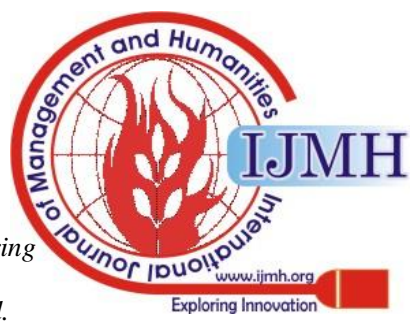


Table 1.4: Interview Findings for Lecturers' Factor

\begin{tabular}{|c|c|c|c|}
\hline $\begin{array}{l}\text { Example of } \\
\text { responses }\end{array}$ & Respondents & Codes & Themes \\
\hline $\begin{array}{l}\text {..lecturers } \\
\text { are not } \\
\text { working to } \\
\text { the } \\
\text { optimum. } \\
\text { They are } \\
\text { weakly } \\
\text { motivated. } \\
\text { Entitlement } \\
\text { are not } \\
\text { responded to } \\
\text { by } \\
\text { management } \\
\text {. Hazard } \\
\text { allowance } \\
\text { poor.. e }\end{array}$ & AC1 AC2 ST3 & $\begin{array}{l}\text { Poor } \\
\text { motivation. }\end{array}$ & $\begin{array}{l}\text { Dampened } \\
\text { morale }\end{array}$ \\
\hline $\begin{array}{l}\text { The } \\
\text { composure } \\
\text { of most } \\
\text { lecturers are } \\
\text { not } \\
\text { good...they } \\
\text { shows too } \\
\text { much ego in } \\
\text { teaching...s } \\
\text { ome are very } \\
\text { good while } \\
\text { some are not } \\
\text { good }\end{array}$ & $\begin{array}{l}\text { AC1 AC2 ST1 } \\
\text { ST2 ST3 }\end{array}$ & $\begin{array}{l}\text { Relationshi } \\
\text { p with } \\
\text { students }\end{array}$ & $\begin{array}{l}\text { Lecturer } \\
\text { hostility }\end{array}$ \\
\hline $\begin{array}{l}\text { The } \\
\text { equipment, } \\
\text { machines } \\
\text { and tools are } \\
\text { obsolete...so } \\
\text { me are not } \\
\text { functioning } \\
\text {...the } \\
\text { available are } \\
\text { not } \\
\text { functioning } \\
\text {...funds not } \\
\text { release for } \\
\text { consumable } \\
\text { materials }\end{array}$ & $\begin{array}{l}\text { AC1 AC2 } \\
\text { AC3 ST2 ST3 }\end{array}$ & $\begin{array}{l}\text { Absence of } \\
\text { teaching } \\
\text { material } \\
\text { for } \\
\text { workshop } \\
\text { practice. }\end{array}$ & $\begin{array}{l}\text { Lack of } \\
\text { materials/ } \\
\text { tools for } \\
\text { practical } \\
\text { teaching. }\end{array}$ \\
\hline $\begin{array}{l}\text {...most of } \\
\text { the course in } \\
\text { TVET are } \\
\text { not relevant } \\
\text { to the future } \\
\text { need of the } \\
\text { student...too } \\
\text { many } \\
\text { courses } \\
\text { offering }\end{array}$ & $\begin{array}{l}\text { AC1 AC2 } \\
\text { AC3 ST1 ST2 } \\
\text { ST3 }\end{array}$ & $\begin{array}{l}\text { Overloade } \\
\text { d } \\
\text { curriculum }\end{array}$ & $\begin{array}{l}\text { Overloade } \\
\mathrm{d} \\
\text { curriculum }\end{array}$ \\
\hline
\end{tabular}

Table 1.5: Summary of Interview Findings for Lectures Factor

\begin{tabular}{|l|c|c|c|c|c|c|c|c|c|c|}
\hline Sub & \multicolumn{4}{|l|}{ Academics } & \multicolumn{5}{|c|}{ Students } \\
\cline { 2 - 9 } Construct & A & A & A & A & A & S & S & S & S & S \\
& C & C & C & C & C & T & T & T & T & T \\
& 1 & 2 & 3 & 4 & 5 & 1 & 2 & 3 & 4 & 5 \\
\hline $\begin{array}{l}\text { Dampened } \\
\text { morale }\end{array}$ & $*$ & $*$ & & & & & & $*$ & & \\
\hline $\begin{array}{l}\text { Lecturers } \\
\text { hostility }\end{array}$ & $*$ & $*$ & & & & $*$ & $*$ & $*$ & & \\
\hline $\begin{array}{l}\text { Lack of } \\
\text { materials } \\
\text { and tools }\end{array}$ & $*$ & $*$ & & & & & $*$ & $*$ & & \\
\hline $\begin{array}{l}\text { Overloaded } \\
\text { curriculum }\end{array}$ & $*$ & $*$ & $*$ & & & $*$ & $*$ & $*$ & & \\
& & & & & & & & & & \\
\hline
\end{tabular}

Table 1.6: Interview Findings for College Factor

\begin{tabular}{|c|c|c|c|}
\hline $\begin{array}{l}\text { Example of } \\
\text { responses }\end{array}$ & $\begin{array}{l}\text { Respond } \\
\text { ents }\end{array}$ & Codes & Themes \\
\hline $\begin{array}{l}\text { The equipment, } \\
\text { machines and } \\
\text { tools are } \\
\text { obsolete...some } \\
\text { are not } \\
\text { functioning...the } \\
\text { available are not } \\
\text { functioning...fun } \\
\text { ds not release for } \\
\text { consumable } \\
\text { materials... } \\
\text { teaching on only } \\
\text { done } \\
\text { theoretically. }\end{array}$ & $\begin{array}{l}\text { AC1 AC2 } \\
\text { AC3 ST1 } \\
\text { ST2 ST3 }\end{array}$ & $\begin{array}{l}\text { Absenc } \\
\text { e of } \\
\text { teachin } \\
\text { g } \\
\text { material } \\
\text { for } \\
\text { worksh } \\
\text { op } \\
\text { practice } \\
\text {. }\end{array}$ & $\begin{array}{l}\text { Teaching } \\
\text { materials/ } \\
\text { tools. }\end{array}$ \\
\hline $\begin{array}{l}\text {...most of the } \\
\text { course in TVET } \\
\text { are not relevant to } \\
\text { the future need of } \\
\text { the student...too } \\
\text { many courses } \\
\text { offering. } \\
\text { Assignments } \\
\text { overload affect } \\
\text { students } \\
\text { performance. }\end{array}$ & $\begin{array}{l}\text { AC1 AC2 } \\
\text { AC3 ST1 } \\
\text { ST2 ST3 }\end{array}$ & $\begin{array}{l}\text { Overloa } \\
\text { ded } \\
\text { curricul } \\
\text { um }\end{array}$ & $\begin{array}{l}\text { Overload } \\
\text { ed } \\
\text { curriculu } \\
\text { m }\end{array}$ \\
\hline $\begin{array}{l}\text { Failure to } \\
\text { maintain stable } \\
\text { academic } \\
\text { calender...many } \\
\text { atimes, two } \\
\text { months makes a } \\
\text { semester... } \\
\text { Failure to } \\
\text { maintain stable } \\
\text { academic } \\
\text { calender...many } \\
\text { atimes, two } \\
\text { months makes a } \\
\text { semester.. }\end{array}$ & $\begin{array}{l}\text { AC2 AC3 } \\
\text { STI ST2 } \\
\text { ST3 }\end{array}$ & $\begin{array}{l}\text { Unstabl } \\
\text { e } \\
\text { academi } \\
\text { c } \\
\text { calenda } \\
\text { r }\end{array}$ & $\begin{array}{l}\text { Academic } \\
\text { calendar }\end{array}$ \\
\hline
\end{tabular}


Failure Factors Quality in Vocational and Technical Education in Nigeria Higher Institutions: The Use of Rasch Model

\begin{tabular}{|c|c|c|c|}
\hline $\begin{array}{l}\text { No teaching and } \\
\text { instructional } \\
\text { material for } \\
\text { workshop } \\
\text { practice...this } \\
\text { made teaching to } \\
\text { be dogmatic and } \\
\text { theoretical... }\end{array}$ & $\begin{array}{l}\text { AC1 AC2 } \\
\text { ST2 }\end{array}$ & $\begin{array}{l}\text { Failure } \\
\text { to use } \\
\text { varied } \\
\text { teachin } \\
\text { g } \\
\text { method } \\
\text { s/ } \\
\text { techniq } \\
\text { ues. }\end{array}$ & $\begin{array}{l}\text { Teaching } \\
\text { material }\end{array}$ \\
\hline $\begin{array}{l}\text { The learning } \\
\text { environment is } \\
\text { grossly } \\
\text { uninviting...it } \\
\text { does not } \\
\text { encourage } \\
\text { learning to } \\
\text { stimulate } \\
\text { interest...I think } \\
\text { the environment is } \\
\text { not too pleasant } \\
\text { for teaching/ } \\
\text { learning the } \\
\text { environment is } \\
\text { not seren...the } \\
\text { road network is } \\
\text { not good. }\end{array}$ & $\begin{array}{l}\text { AC1 AC2 } \\
\text { AC3 ST1 } \\
\text { ST2 ST3 }\end{array}$ & $\begin{array}{l}\text { Poor } \\
\text { learning } \\
\text { environ } \\
\text { ment }\end{array}$ & $\begin{array}{l}\text { Aesthetic } \\
\mathrm{s}\end{array}$ \\
\hline $\begin{array}{l}\text { Non-payment of } \\
\text { school fees } \\
\text { normally leads to } \\
\text { outstanding and } \\
\text { failure...the } \\
\text { school fees is too } \\
\text { high... we are not } \\
\text { given grace to } \\
\text { complete } \\
\text { payment...the } \\
\text { present Provost is } \\
\text { trying... }\end{array}$ & $\begin{array}{l}\text { AC3 ST1 } \\
\text { ST2 ST3 }\end{array}$ & $\begin{array}{l}\text { Burden } \\
\text { of high } \\
\text { tuition }\end{array}$ & $\begin{array}{l}\text { High } \\
\text { tuition }\end{array}$ \\
\hline $\begin{array}{l}\text { We are always } \\
\text { faced with } \\
\text { registration } \\
\text { problem...remitta } \\
\text { challenge }\end{array}$ & $\begin{array}{l}\text { ST1 ST2 } \\
\text { ST3 }\end{array}$ & $\begin{array}{l}\text { Student } \\
\text { registrat } \\
\text { ion }\end{array}$ & $\begin{array}{l}\text { Hardship } \\
\text { in } \\
\text { registratio } \\
\text { n }\end{array}$ \\
\hline $\begin{array}{l}\text { The } \\
\text { infrastructures } \\
\text { enjoyed by other } \\
\text { students in other } \\
\text { institution are not } \\
\text { present in the } \\
\text { College...no wifi, } \\
\text { power supply, } \\
\text { water and so } \\
\text { on...no } \\
\text { scholarship to } \\
\text { student. }\end{array}$ & ST2 ST3 & $\begin{array}{l}\text { Absenc } \\
\text { e of } \\
\text { infrastr } \\
\text { ucture }\end{array}$ & $\begin{array}{l}\text { Social } \\
\text { amenities }\end{array}$ \\
\hline
\end{tabular}

Table 1.7: Summary of Interview Findings for College Factor

\begin{tabular}{|c|c|c|c|c|c|c|c|c|c|c|}
\hline \multirow[t]{4}{*}{ Sub Construct } & \multicolumn{5}{|c|}{ Academics } & \multicolumn{5}{|c|}{ Students } \\
\hline & $\mathrm{A}$ & $\mathrm{A}$ & A & $\mathrm{A}$ & $\mathrm{A}$ & $\mathrm{S}$ & $\mathrm{S}$ & $\mathrm{S}$ & $\mathrm{S}$ & $\mathrm{S}$ \\
\hline & $\mathrm{C}$ & $\mathrm{C}$ & $\mathrm{C}$ & $\mathrm{C}$ & $\mathrm{C}$ & $\mathrm{T}$ & $\mathrm{T}$ & $\mathrm{T}$ & $\mathrm{T}$ & $\mathrm{T}$ \\
\hline & 1 & 2 & 3 & 4 & 5 & 1 & 2 & 3 & 4 & 5 \\
\hline $\begin{array}{l}\text { Teaching } \\
\text { materials/tools }\end{array}$ & $*$ & $*$ & $*$ & & & $*$ & $*$ & $*$ & & \\
\hline $\begin{array}{l}\text { Overloaded } \\
\text { curriculum }\end{array}$ & $*$ & $*$ & $*$ & & & $*$ & $*$ & $*$ & & \\
\hline $\begin{array}{l}\text { Unstable } \\
\text { academic } \\
\text { calendar }\end{array}$ & & $*$ & $*$ & & & $*$ & $*$ & $*$ & & \\
\hline $\begin{array}{l}\text { Absence of } \\
\text { Teaching } \\
\text { materials }\end{array}$ & $*$ & $*$ & & & & & $*$ & & & \\
\hline Aesthetics & $*$ & $*$ & $*$ & & & $*$ & $*$ & $*$ & & \\
\hline High tuition & & & $*$ & & & $*$ & $*$ & $*$ & & \\
\hline $\begin{array}{l}\text { Hardship in } \\
\text { registration }\end{array}$ & & & & & & $*$ & $*$ & $*$ & & \\
\hline $\begin{array}{l}\text { Social } \\
\text { amenities }\end{array}$ & & & & & & & $*$ & $*$ & & \\
\hline
\end{tabular}

Table 1.8: Interview Findings for Government Factor

\begin{tabular}{|l|l|l|l|}
\hline $\begin{array}{l}\text { Example of } \\
\text { responses }\end{array}$ & $\begin{array}{l}\text { Respond } \\
\text { ents }\end{array}$ & Codes & Themes \\
\hline $\begin{array}{l}\text { Government needs to } \\
\text { fund TVET } \\
\text { adequately...technical } \\
\text { education is relegated } \\
\text { to the } \\
\text { background...need for } \\
\text { the provision of } \\
\text { educational resources } \\
\text { in schools. }\end{array}$ & $\begin{array}{l}\text { AC2 ST1 } \\
\text { ST2 }\end{array}$ & $\begin{array}{l}\text { Poor } \\
\text { fundin } \\
\text { g of } \\
\text { educati } \\
\text { on }\end{array}$ & $\begin{array}{l}\text { Inadequate } \\
\text { ly funding }\end{array}$ \\
\hline $\begin{array}{l}\text { Most of us are in the } \\
\text { College by } \\
\text { chance...We actually } \\
\text { want to go to the } \\
\text { university....just } \\
\text { because we are poor. } \\
\text { That is why...no one } \\
\text { want to be a teacher. }\end{array}$ & ST2 ST3 & $\begin{array}{l}\text { AC1 } \\
\text { percept } \\
\text { ion of } \\
\text { TVET/ } \\
\text { Colleg }\end{array}$ & \\
\hline & & $\begin{array}{l}\text { e of } \\
\text { educati } \\
\text { on }\end{array}$ & \\
\hline
\end{tabular}

Table 1.9: Summary of Interview Findings for Government Factor

\begin{tabular}{|l|c|c|c|c|c|c|c|c|c|c|}
\hline Sub & \multicolumn{9}{|l|}{ Academics } & \multicolumn{6}{|c|}{ Students } \\
\cline { 2 - 9 } Construct & A & A & A & A & A & S & S & S & S & S \\
& C & C & C & C & C & T & T & T & T & T \\
& 1 & 2 & 3 & 4 & 5 & 1 & 2 & 3 & 4 & 5 \\
\hline $\begin{array}{l}\text { Inadequate } \\
\text { funding }\end{array}$ & $*$ & $*$ & & & & $*$ & $*$ & & & \\
\hline $\begin{array}{l}\text { Image of } \\
\text { teaching } \\
\text { profession }\end{array}$ & $*$ & $*$ & & & & $*$ & $*$ & $*$ & & \\
\hline
\end{tabular}

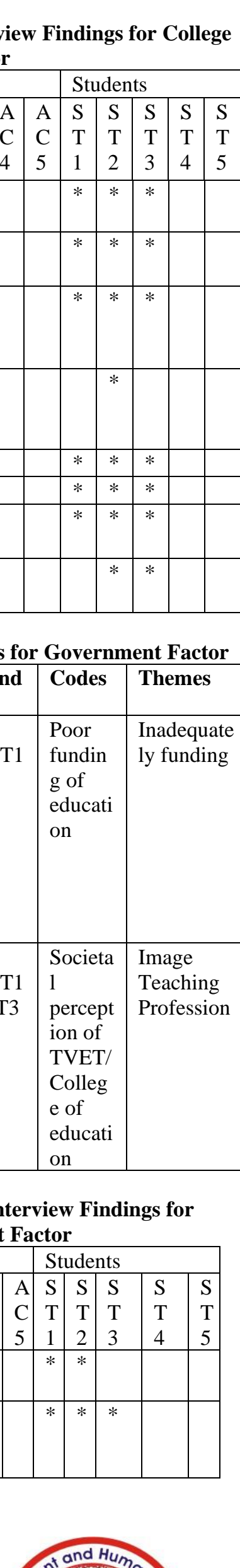
.
Published By:

Blue Eyes Intelligence Engineering

\& Sciences Publication

(C) Copyriaht: All riahts reserved. 


\section{RESULTS}

The result of the initial finding (qualitative) is shown in table 1.10 .

Table 1.10: Initial Factors Responsible for Students Failure in TVET

\begin{tabular}{|c|c|c|}
\hline \\
\hline \multicolumn{3}{|c|}{$\begin{array}{l}\text { INTERNAL FACTOR } \\
\text { STUDENTS FACTOR } \\
\text {. Absenteeism } \\
\text {. High tuition } \\
\text {. Lack of motivation } \\
\text {. Uncared attitudes } \\
\text {. Abstract teaching }\end{array}$} \\
\hline \multicolumn{3}{|c|}{ EXTERNAL FACTOR } \\
\hline $\begin{array}{l}\text { LECTURER } \\
\text { FACTOR } \\
\text {. Dampened } \\
\text { morale } \\
\text {. Hostility to } \\
\text { students } \\
\text {. lack of } \\
\text { material for } \\
\text { practical }\end{array}$ & $\begin{array}{l}\text { COLLEGEFACTOR } \\
\text {. Teaching materials/ } \\
\text { tools } \\
\text {. Overloaded } \\
\text { curriculum } \\
\text {. unstable Academic } \\
\text { calendar } \\
\text {. Aesthetics } \\
\text {. High tuition } \\
\text {. Hardship in } \\
\text { registration } \\
\text {. Absence of social } \\
\text { amenities }\end{array}$ & $\begin{array}{l}\text { GOVERNMENT } \\
\text { FACTOR } \\
\text {. Inadequate } \\
\text { funding } \\
\text {. Poor image of } \\
\text { teaching } \\
\text { profession }\end{array}$ \\
\hline
\end{tabular}

The word view of the constructs is shown is Figure 1.2 as generated using NVIVO 12 software.

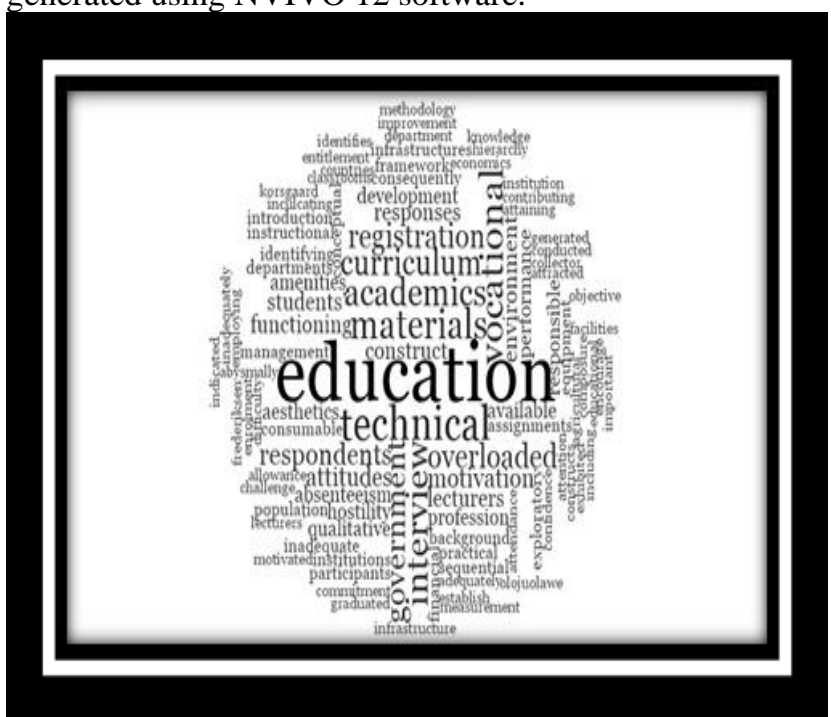

Figure 1.2: Word View.

SUMMARY OF 36 MEASURED ITEM

\begin{tabular}{|c|c|c|c|c|}
\hline | & TOTAL & & \multirow{4}{*}{ MEASURE } & MODEI \\
\hline INEIT & OUTEIT & | & & \multirow{3}{*}{ ERROR } \\
\hline 1 & SCORE & COUNT & & \\
\hline MNSQ & MNSQ & ZSTD & & \\
\hline --ー-- & $\begin{array}{l}------ \\
------\end{array}$ & & & \\
\hline $\begin{array}{l}\text { MEAN } \\
4.33\end{array}$ & $5.8^{120.6} 7.46$ & $\begin{array}{l}40.0 \\
7.9\end{array}$ & .00 & \\
\hline
\end{tabular}

\begin{tabular}{lccccc} 
S.D. & \multicolumn{2}{c}{8.7} & .0 & 1.74 & .05 \\
3.25 & 2.6 & 2.75 & 2.0 & & \\
| MAX. & 144.0 & 40.0 & 1.63 & .22 \\
9.90 & 9.9 & 9.90 & $9.9 \mid$ & & \\
| MIN. & 95.0 & 40.0 & -3.01 & .10 \\
1.55 & 2.0 & 2.79 & 3.9 & &
\end{tabular}

I REAT RMSE

5.38 ITEM RELIABILITY .97

IMODEL RMSE $\quad .18$ TRUE SD 1.73 SEPARATION 9.44 ITEM RELIABILITY .99

I S.E. OF ITEM MEAN $=.29$

$\mathrm{UMEAN}=.0000 \mathrm{USCALE}=1.0000$

TEM RAW SCORE-TO-MEASURE CORRELATION $=-.76$ 1440 DATA POINTS. LOG-LIKELIHOOD CHI-SQUARE: 9180.43 with 1324 d.f. $p=.0000$

Global Root-Mean-Square Residual (excluding extreme scores): 10.3691

Figure 1.3: Measured Item

35. OVC3

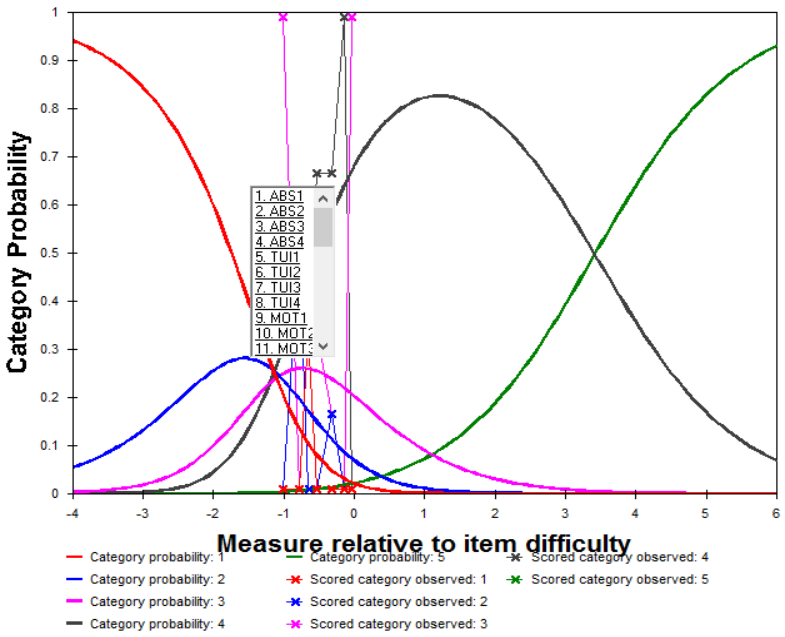

Figure 1.4: ICC Plots Category

Table 1.11: Expert consensus model

\begin{tabular}{|l|l|l|l|}
\hline \multicolumn{4}{|l|}{ INTERNAL FACTOR } \\
\hline Variable & Value & Variable & Value \\
\hline ABS1 & -1.04 & ABS3 & .21 \\
\hline ABS2 & -.25 & ABS4 & .00 \\
\hline TUI1 & -.56 & TUI2 & .21 \\
\hline TUI3 & -.25 & MOT1 & .00 \\
\hline TUI4 & -.56 & MOT2 & .21 \\
\hline EXTERNAL FACTOR & \multicolumn{1}{l|}{} \\
\hline MOT4 & -.56 & MOT3 & .21 \\
\hline STA3 & -1.04 & STA1 & .21 \\
\hline STA4 & -.25 & STA2 & .41 \\
\hline ABT3 & -.56 & ABT1 & .21 \\
\hline TOM2 & -1.04 & ABT2 & .00 \\
\hline OVC2 & -1.04 & ABT4 & .21 \\
\hline OVC4 & -.27 & DMO1 & .00 \\
\hline & & DM02 & .21 \\
\hline & & DMO3 & .21 \\
\hline & & DMO4 & .84 \\
\hline & & LEA1 & .21 \\
\hline
\end{tabular}

Blue Eyes Intelligence Engineering

\& Sciences Publication

(C) Copyriaht: All riahts reserved. 
Failure Factors Quality in Vocational and Technical Education in Nigeria Higher Institutions: The Use of Rasch Model

\begin{tabular}{|l|l|l|l|}
\hline & & LEA2 & .21 \\
\hline & & LEA3 & .52 \\
\hline & & LEA4 & .04 \\
\hline & & TOM1 & .41 \\
\hline & & TOM3 & .41 \\
\hline & & TOM4 & .84 \\
\hline & & OVC1 & .00 \\
\hline & & OVC3 & .00 \\
\hline
\end{tabular}
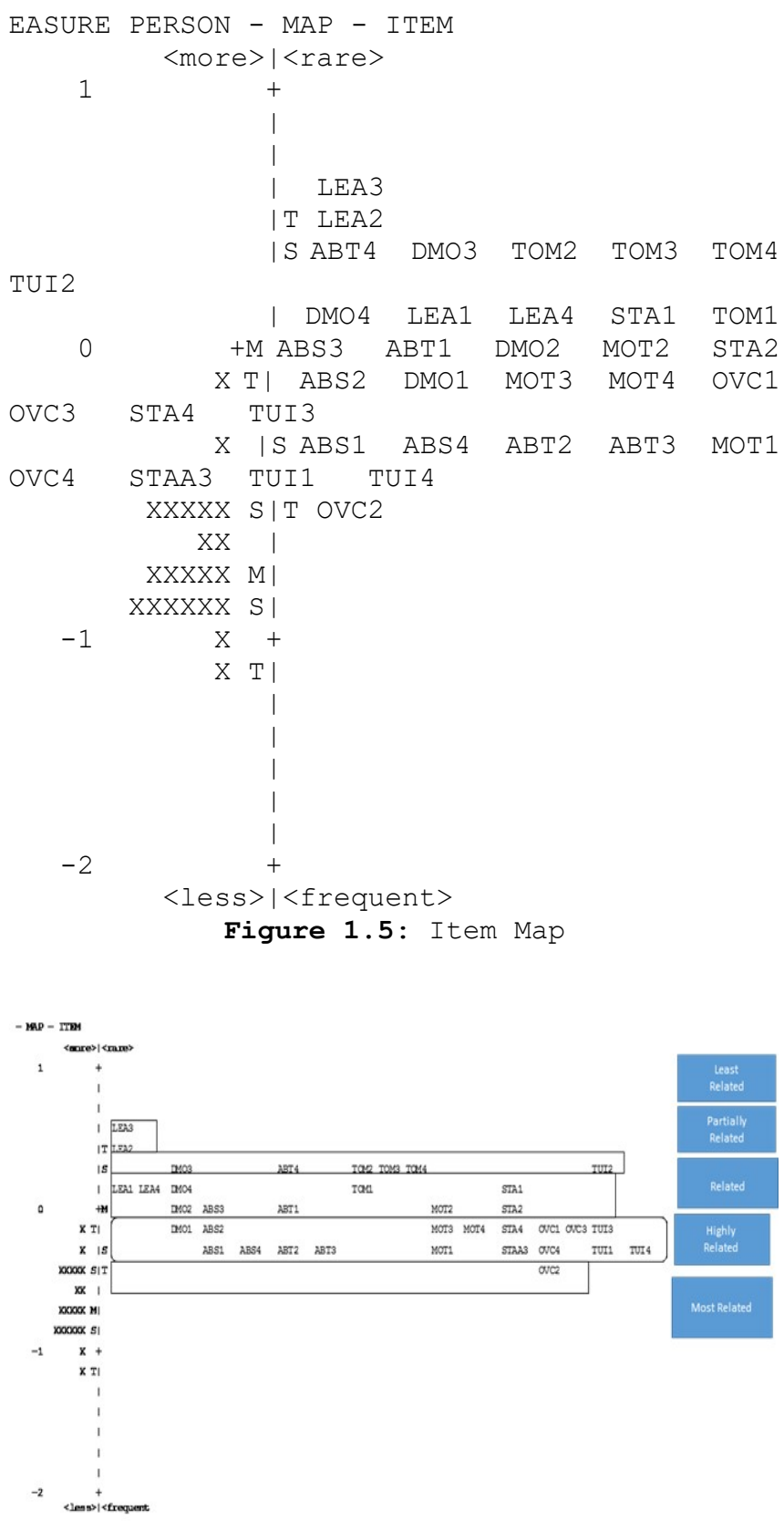

Figure 1.6: Item Hierarchy of Relativity and Difficulty Level.

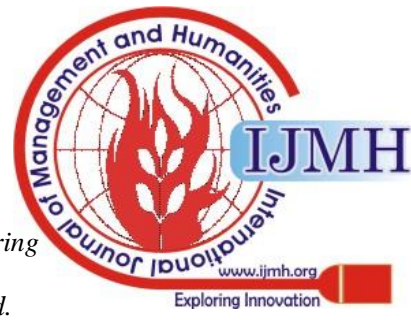


3. E. V. Abolo, "Programs in Nigeria Higher Institutions and Graduate Unemployment," J. Res. Method Educ., vol. 7, no. 2, pp. 1-6, 2016.

4. Federal Government of Nigeria, "National Policy on Education." Abuja, Nigeria, 2013.

5. R. P. S. O. Uddin, "The Role of Technical and Vocational Education in Poverty Reduction among Youths in Nigeria," vol. 4, no. 4, p. 617, 2013.

6. E. S. Tunde, "Graduate Unemployment in Nigeria : Causes, Effects and," Br. J. Arts Soc. Sci., vol. 5, no. 2, pp. 142-154, 2012.

7. M. A. Peters, "Technological Unemployment: Educating for the Fourth Industrial Revolution,” Educ. Philos. Theory, vol. 49, no. 1, pp. 1-6, 2017.

8. A. Otaigbe, "Evaluation of Vocational Education in Nigeria: A Review of the Roles of the Regulatory Bodies National University Commission ( NUC ) The Main Functions of the NUC," vol. 4, no. 3, pp. 16-21, 2015.

9. R. Okoye and M. O. Arimonu, "Technical and Vocational Education in Nigeria : Issues, Challenges and a Way Forward," J. Educ. Pract., vol. 7, no. 3, pp. 113-118, 2016.

10. S. R. Olojuolawe, F. Bt, M. Amin, and M. G. Adewumi, "IR4 . 0 : The Challenges for Graduate Employability in Nigeria," vol. 9, no. 1, pp. 1-5, 2019.

11. M. Lin, "Challenges and Opportunities for Technical and Vocational Education and Training in the Local Communities: Education and Labour Market for Young People,” Int. J. Soc. Sci. Stud., vol. 7, no. 3, p. 1, 2019.

12. N. G. Hayatu and B. B. Abubakar, "Causes of Examination Failure among the Students of Tertiary Institutions in Nigeria : A Review," vol III, no. Vii, pp. 381-384, 2019

13. D. R. Forsyth, P. A. Story, K. N. Kelley, and J. H. McMillan, "What Causes Failure and Success? Students' Perceptions of their Academic Outcomes," Soc. Psychol. Educ., vol. 12, no. 2, pp. 157-174, 2009.

14. P. Blenker, S. T. Elmholdt, S. H. Frederiksen, S. Korsgaard, and K. Wagner, "Methods in Entrepreneurship Education Research: A Review and Integrative Framework," Educ. + Train., vol. 56, no. 8/9, pp. 697-715, 2014.

15. J. W. Creswell, Research Design: Qualitative, Quantitative and Mixed Methods Approaches, Fourth. london,UK., 2013.

16. D. Collector and F. G. Module, "Qualitative Research Methods Overview," Fam. Heal. Int., no. January, pp. 1-12, 2011.

17. C. Anderson, "Presenting and Evaluating Qualitative Research Strengths and Limitations of Qualitative Research," Am. J. Pharm. Educ., vol. 74, no. 8, pp. 1-7, 2010.

18. C. G. Green, K. E., \& Frantom, "Survey Development and Validation with the Rasch Model," in International Conference on Questionnaire Development, Evaluation and Testing, 2002, pp. 1-42.

19. M. Saidfudin, A. . Azrilah, N. A. Rodzo'an, M. . Omar, A. Zaharim, and H. Basri, "Use of Rasch Analysis to Measure Students Performance in Engineering Education," Proc. 7th WSEAS Int. Conf. Eng. Educ. World Sci. Eng. Acad. Soc., pp. 435-441, 2010.

\section{AUTHORS PROFILE}

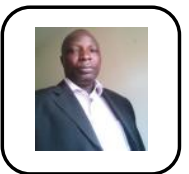

Dr. Olojuolawe, R.S, is a Chief Lecturer at the College of Education, Ikere-Ekiti, Nigeria. He had his doctoral degree at the famous Universiti Teknologi Malaysia. He was the Vice Dean of the School of Vocational and Technical Education, College of Education, Ikere-Ekiti between 2013 and 2015. He had to his credit many publications in both local and international journals. Dr. Olojuolawe completed his undergrute and master degrees in Nigeria. He is happily married.

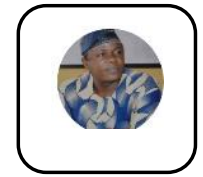

Mr. Tunde Ajayi, is a Chief Lecturer at the College of Education, Ikere-Ekiti, Nigeria. As a seasoned administrator and academics, he had been head of department of Fine and Applied Arts, Dean School of Vocational and Technical Education, and currently the Director of Pre-Degree and Advance studies at the College with many publication to his credit. His marriage is blessed with children.

Mr. Awolumate Emmanuel Olorunfemi, is a lecturer in the department of vocational and technical education college of education Ikere Ekiti. His area of specialisation is electrical/ electronics. He presented candidates for West Africa Examination Council in applied electricity and basic electronics while serving as tutor in senior secondary school. He has published many articles in reputable journals.

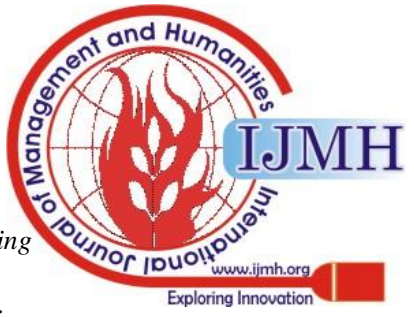

